Check for updates

Cite this: Phys. Chem. Chem. Phys., 2018, 20, 22517

Received 9th June 2018

Accepted 14th August 2018

DOI: $10.1039 / c 8 c p 03655 a$

rsc.li/pccp

\title{
Viscous interfacial layer formation causes electroosmotic mobility reversal in monovalent electrolytes $\dagger$
}

\author{
Majid Rezaei, ${ }^{\text {ab }}$ Ahmad Reza Azimian, ${ }^{a}$ Ahmad Reza Pishevar ${ }^{a}$ and \\ Douwe Jan Bonthuis (iD *b
}

\begin{abstract}
We study the ion density, shear viscosity and electroosmotic mobility of an aqueous monovalent electrolyte at a charged solid surface using molecular dynamics simulations. Upon increasing the surface charge density, ions are displaced first from the diffuse layer to the outer Helmholtz layer, increasing its viscosity, and subsequently to the hydrodynamically stagnant inner Helmholtz layer. The ion redistribution causes both charge inversion and reversal of the electroosmotic mobility. Because of the surface-charge dependent interfacial hydrodynamic properties, however, the charge density of mobility reversal differs from the charge density of charge inversion, depending on the salt concentration and the chemical details of the ions and the surface. Mobility reversal cannot be described by an effective slip boundary condition alone - the spatial dependence of the viscosity is essential.
\end{abstract}

\section{Introduction}

Charged surfaces in solution are neutralized by counterions, forming an electric double layer. Early models of the structure of the double layer have evolved from the compact layer model of Helmholtz, ${ }^{1}$ via the diffuse layer of Gouy and Chapman, ${ }^{2,3}$ to the model of Stern, ${ }^{4}$ where the counterions are distributed over a compact and a diffuse layer. In the Stern model, ions can approach the surface up to a distance of one ionic radius, which means that the solvent is effectively treated as a homogeneous medium and the presence of hydration shells is neglected. Close to the surface, however, the solvent is not homogeneous, and the distance of closest approach of the ions depends on the surface charge density, the interfacial molecular structure and the ionic hydration level. In particular, ions can partly loose their hydration shell under the influence of the surface, allowing the ions to adsorb directly onto the surface. This leads to Grahame's definition of the inner Helmholtz plane (IHP), passing through the centers of the partially dehydrated and tightly adsorbed ions, and the outer Helmholtz plane (OHP), passing through the centers of the hydrated ions at their distance of closest approach to the surface. ${ }^{5}$ The importance of the molecular solvent structure for the macroscopic interfacial properties is corroborated by molecular dynamics simulations. ${ }^{6}$

\footnotetext{
${ }^{a}$ Department of Mechanical Engineering, Isfahan University of Technology,

8415683111 Isfahan, Iran. E-mail: Majid-rezaei@me.iut.ac.ir

${ }^{b}$ Fachbereich Physik, Freie Universität Berlin, 14195 Berlin, Germany.

E-mail: d.j.bonthuis@fu-berlin.de

$\dagger$ Electronic supplementary information (ESI) available. See DOI: 10.1039/c8cp03655a
}

The prevalent method used to measure the surface charge density of solutes is through electrokinetic experiments. Upon application of an electric field, stress generated in the electric double layer gives rise to motion of the fluid with respect to the surface. Relying on high surface-to-volume ratios, this electroosmotic flow is of particular interest in the context of gel electrophoresis, ${ }^{7}$ nano and microfluidics, lab-on-a-chip devices ${ }^{8}$ and surface characterization, ${ }^{9}$ but also for biological function ${ }^{10}$ and drug delivery. ${ }^{11}$ At low surface charge density, the electroosmotic mobility increases linearly with the surface charge density, allowing for a straightforward measurement. At higher surface charge density, the dependence of the mobility on the surface charge turns into a power law, caused by the double-layer structure. ${ }^{12,13}$ Comparing with experiments on hydrophilic and hydrophobic colloids, the power law has been verified to be valid up to $\sim 0.1 \mathrm{C} \mathrm{m}^{-2}$. Many solutes, however, carry higher surface charge densities, such as DNA $(2 e / 0.34 \mathrm{~nm}, 1 \mathrm{~nm}$ radius: $\sim 0.15 \mathrm{C} \mathrm{m}^{-2}$ ) and many proteins (average surface charge density of up to $0.3 \mathrm{C} \mathrm{m}^{-214}$ ). The macroscopic dynamics of such highly charged objects in water are strongly affected by the hydrodynamic properties of the hydration layer. ${ }^{15}$

At high surface charge density, ions form a correlated liquid in the close vicinity of the charged surface, depending on the salt concentration, ion valency and the lateral distribution of the surface charges, ${ }^{16}$ which cannot be described by classical mean-field theory. ${ }^{17}$ These correlations can lead to charge inversion, meaning that in a thin layer close the surface, the ionic counter charge density exceeds the surface charge density, whereas the rest of the fluid carries a net charge of the same 
sign as the surface charge. AFM measurements of charge inversion confirm that the charge inversion is caused primarily by correlations between the counterions. ${ }^{18}$ Assuming the interfacial layer, which is typically identified with the Stern layer, to be hydrodynamically stagnant, charge inversion causes reversal of the electrophoretic mobility. Such mobility reversal has been observed for many different systems, ${ }^{16}$ also for monovalent ions at colloids ${ }^{19,20}$ and inside protein channels. ${ }^{21}$ Unlike charge inversion, ${ }^{18}$ however, mobility reversal depends not only on the ion valency but also strongly on the chemical details of the substances involved. ${ }^{19-21}$ Moreover, the point of mobility reversal does not generally coincide with the point of charge inversion estimated from coagulation experiments, ${ }^{22}$ and ionion correlations are insufficient for a quantitative description of mobility reversal. ${ }^{23}$ Crucial for the interpretation of these measurements is the question how the presence of the surface charge and the ions affect the interfacial water structure and its hydrodynamic properties, ${ }^{24,25}$ which has not been answered so far.

In this paper, we study the interfacial ion distribution as a function of the surface charge density and its effect on the electroosmotic mobility and shear velocity profile. We show that charge inversion between the two Helmholtz layers and the diffuse layer occurs at a surface charge density which depends on the salt concentration and the lateral distribution of the surface charge, but also on the size and interaction strength of the counterions. In the charge-inverted systems, also the electroosmotic mobility changes sign, but not at the surface charge density at which charge inversion takes place. The reason for this discrepancy is the fact that whereas the inner Helmholtz layer is immobile relative to the surface, the viscosity of the outer Helmholtz layer remains finite, and depends strongly on the ion concentration and surface charge density.

\section{Methods}

\section{Simulation details}

We simulate an aqueous solution of $\mathrm{NaCl}$ confined between two parallel solid walls (Fig. 1). Water is modeled using the SPC/E model $^{26}$ and ions are modeled as charged Lennard-Jones (LJ) spheres. For the background salt concentration, $\mathrm{N}_{\mathrm{Cl}^{-}} \mathrm{Cl}^{-}$ions are distributed in the solvent, and the number of $\mathrm{Na}^{+}$ions is adjusted to ensure overall charge neutrality. The walls consist of four layers of silicon ( $\mathrm{Si}$ ) atoms arranged in an FCC-lattice with a lattice constant of $a=0.5431 \mathrm{~nm}$, cut in the (111) direction. A fraction $\phi_{q}$ of the surface layers of the solid carry a negative partial charge $q_{\mathrm{i}}$ while the other $\mathrm{Si}$ atoms are electrically neutral, leading to a surface charge density $\Sigma=4 \phi_{q} q_{\mathrm{i}} /\left(\sqrt{3} a^{2}\right)$. The width of the channel, defined as the distance between the surface layers of Si atoms (directly adjacent to the fluid), is $4.84 \mathrm{~nm}$. Starting with a system that we refer to as the standard system (system 1, see Table S1, ESI $\dagger$ ), we also vary the other system parameters, in particular the ion-water interaction, the salt concentration, the electric field strength and the lateral surface charge distribution (systems $2-7$, see Table S1, ESI $\dagger$ ).

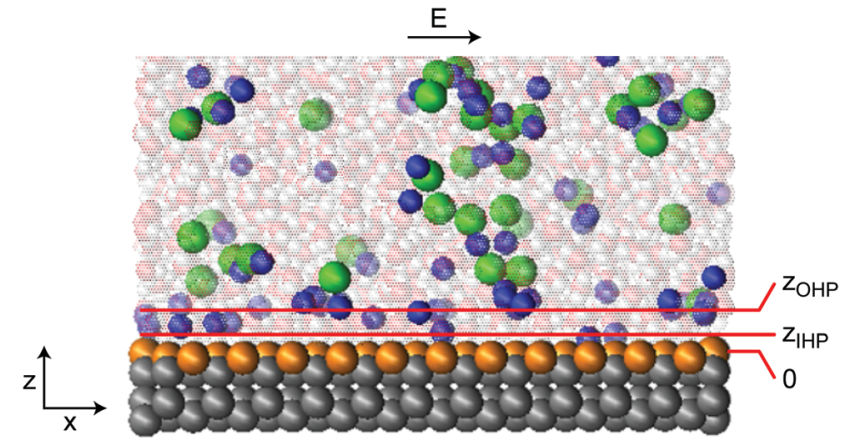

Fig. 1 Schematic view of the standard simulation system with the positions of the outermost surface layer $(z=0)$ and the inner and outer Helmholtz planes, $z_{\mathrm{IHP}}$ and $z_{\mathrm{OHP}}$ indicated. The snapshot shows up to the center of the symmetrical system and blue, green, orange, and silver atoms indicate $\mathrm{Na}^{+}$, $\mathrm{Cl}^{-}$, charged $\mathrm{Si}$, and uncharged $\mathrm{Si}$ atoms, respectively.

In the standard system, $\phi_{q}=1$, meaning that every surface atom carries an equal partial charge and $N_{\mathrm{Cl}^{-}}=80$, corresponding to a background concentration of $1.7 \mathrm{~mol} \mathrm{l}^{-1}$. The number of water molecules is set at 2951 such that the initial pressure is zero. The dimensions of the simulation box are $x \times y \times z=4.66 \times$ $4.50 \times 22.0 \mathrm{~nm}$ and periodic boundary conditions are used in all directions. The purpose of the large box size in $z$ direction is to reduce electrostatic interactions between the periodic images. For the electroosmosis simulations, we apply an external electric field $E_{\|}$parallel to the surface, using $E_{\|}=0.55 \mathrm{~V} \mathrm{~nm}^{-1}$ in the standard system. The shear simulations (system 8, see Table S1, ESI $\dagger$ ) are performed by moving the channel walls in opposite direction with a constant velocity $v_{0}= \pm 50 \mathrm{~m} \mathrm{~s}^{-1}$ and calculating the shear force $F$ on the channel wall. All the shearing simulations are performed in the linear friction regime, which is verified by monitoring the shear-velocity dependence of the results (see Section S2, ESI $\dagger$ ). The pair-wise interaction potential between the atoms $i$ and $j$ is given by

$$
V_{\mathrm{ij}}\left(r_{\mathrm{ij}}\right)=4 \varepsilon_{\mathrm{ij}}\left[\left(\frac{\sigma_{\mathrm{ij}}}{r_{\mathrm{ij}}}\right)^{12}-\left(\frac{\sigma_{\mathrm{ij}}}{r_{\mathrm{ij}}}\right)^{6}\right]+\frac{q_{\mathrm{i}} q_{\mathrm{j}}}{4 \pi \varepsilon_{0} r_{\mathrm{ij}}},
$$

with $r_{\mathrm{ij}}$ being the separation distance between two atoms, $\sigma_{\mathrm{ij}}$ and $\varepsilon_{\mathrm{ij}}$ being the Lennard-Jones size and interaction strength, respectively, $q_{\mathrm{i}, \mathrm{j}}$ being the atomic (partial) charge and $\varepsilon_{0}$ being the vacuum permittivity. For the Lennard-Jones interaction we use a cutoff radius of $1.1 \mathrm{~nm}$. For the long-range Coulomb interactions the $\mathrm{P} 3 \mathrm{M}$ algorithm ${ }^{27}$ is employed, tuned to obtain a maximum relative error of $10^{-4}$ in the calculated forces. System energy minimization is accomplished via the conjugate gradient method and the initial velocity for each atom is determined using a Gaussian distribution based on the specified temperature. The simulations are performed using LAMMPS, ${ }^{28}$ using the velocity Verlet method with a 2 fs time step in the $N V T$ ensemble. Temperature is maintained constant using a velocityrescale algorithm, where only the velocity components in the directions orthogonal to the flow (i.e., $y$ and $z$ components) are adjusted. ${ }^{24}$ In all cases, the system is equilibrated for $2 \mathrm{~ns}$ before running the main simulations for more than $60 \mathrm{~ns}$. 
For the interpretation of our results, it should be kept in mind that the simulations have been performed using ions in pure $\mathrm{H}_{2} \mathrm{O}$. In experimental settings, there is substantial evidence that trace amounts of surfactant from the water, adsorbing strongly on hydrophobic surfaces, render them negatively charged at neutral $\mathrm{pH}^{29,30}$ Based on the saturation of the electrokinetic mobility with decreasing surfactant concentration observed in simulations of airwater interfaces, it has been suggested that even small amounts of surfactants might affect the electrokinetic mobility. ${ }^{31}$ In our simulations, we do not take surfactants into account explicitly; instead, the surface charge $\Sigma$ is considered to include the effect of the adsorbed impurities on the effective surface charge. Nevertheless, the absence of explicit impurities in our simulations might limit the direct applicability of our results to experimental systems.

\section{Results and discussion}

\section{Layered interfacial structure}

In Fig. 2, we show the ion number densities ( $c_{+}$and $c_{-}$, left axis) and the water molecular number density $\left(c_{\mathrm{H}_{2} \mathrm{O}}\right.$, right axis) in the standard system (system 1, see Table S1, ESI $\dagger$ ) at four different values of the surface charge density $\Sigma$. Clearly, the results show the existence of two to three distinct peaks in the ion density profiles.
At low surface charge density (Fig. 2a), counterions are attracted to the interface as expected, but the counterion density is not monotonic. Instead, there are two peaks at $z=0.5 \mathrm{~nm}$ and $z=$ $0.8 \mathrm{~nm}$, both with fully hydrated ions, as can be seen from the water density. Following Grahame, we identify the first hydrated peak with the outer Helmholtz plane (at position $z_{\mathrm{OHP}}$ ) and the other peak with the diffuse layer. We define the peaks in the water density to the left and to the right of $z_{\mathrm{OHP}}$ as the boundaries of the outer Helmholtz layer. When the surface charge density is increased, ions redistribute from the diffuse layer into the outer Helmholtz layer (Fig. 2b). At even higher surface charge density (Fig. 2c and d), a third peak appears, containing ions which are partially dehydrated. We identify this peak with the inner Helmholtz plane (at position $z_{\mathrm{IHP}}$ ). When the surface charge increases, the number of ions in the inner Helmholtz layer increases at the expense of the outer Helmholtz layer. Interestingly, when the inner Helmholtz layer is populated, the system starts exhibiting charge inversion, as can be seen from the displacement field

$$
D_{\perp}(z)=\Sigma+\int_{0}^{z} e\left(c_{+}\left(z^{\prime}\right)-c_{-}\left(z^{\prime}\right)\right) \mathrm{d} z^{\prime}
$$

shown in the insets of Fig. 2, which changes sign for high surface charge densities. The position at which the displacement field
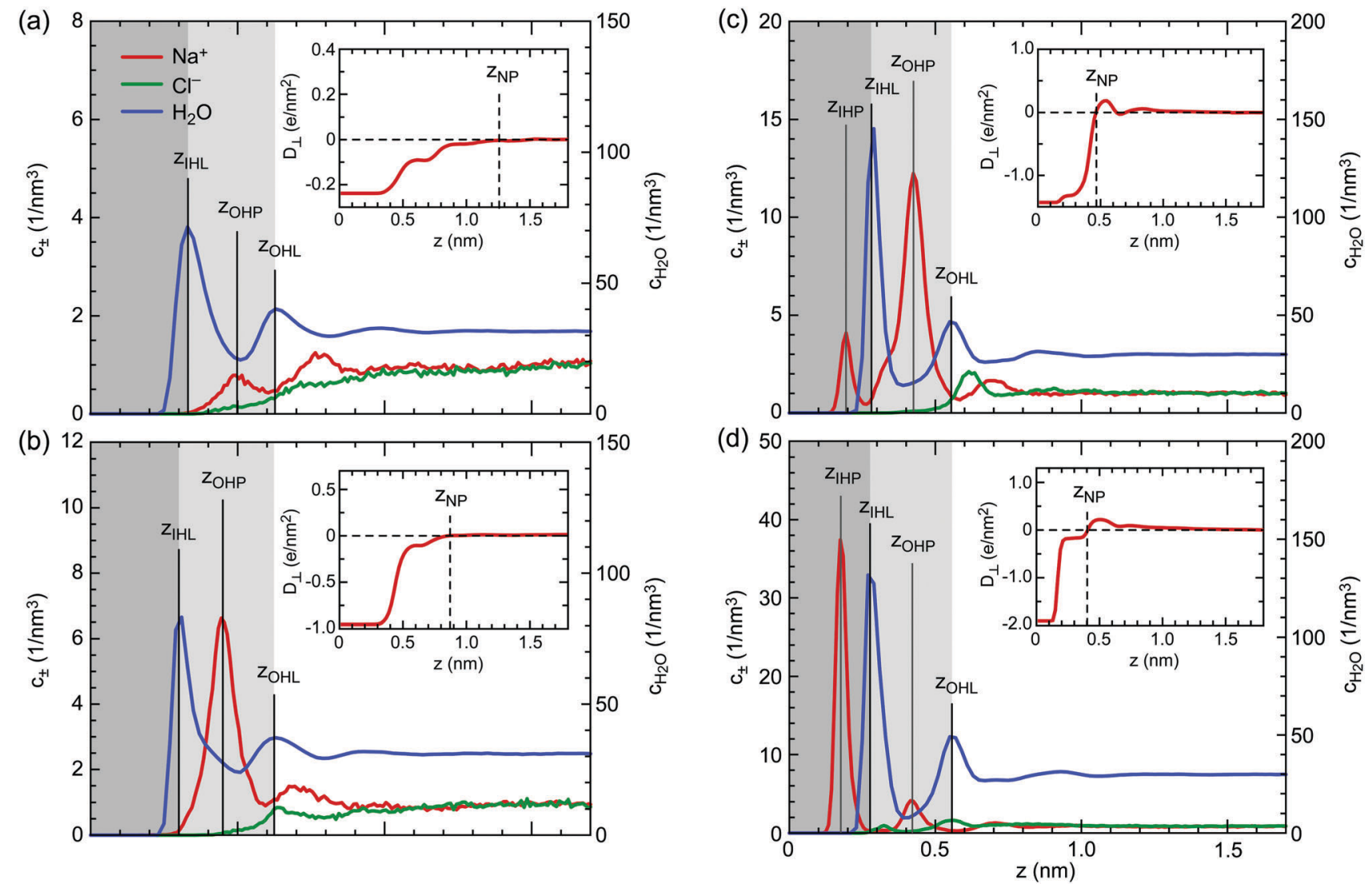

Fig. 2 Ionic $\left(c_{ \pm}\right)$and water $\left(c_{\mathrm{H}_{2} \mathrm{O}}\right)$ molecular number density profiles as a function of the position $z$ for different surface charge densities: (a) $\Sigma=-0.0385 \mathrm{C} \mathrm{m}^{-2}$, (b) $\Sigma=-0.155 \mathrm{C} \mathrm{m}^{-2}$, (c) $\Sigma=-0.23 \mathrm{C} \mathrm{m}^{-2}$ and (d) $\Sigma=-0.308 \mathrm{C} \mathrm{m}^{-2}$. The positions of the inner ( $z_{\mathrm{IHP}}$ ) and outer ( $z_{\mathrm{OHP}}$ ) Helmholtz planes are indicated by vertical lines, together with the boundaries of the inner and outer Helmholtz layers $\left(z_{1 H L}, z_{O H L}\right)$, defined as the positions of the water peaks separating the inner and outer Helmholtz planes and the diffuse layer. The insets show the perpendicular displacement field (eqn (2)) and the location of the charge neutral plane, $z_{\mathrm{NP}}$, where the displacement field first vanishes. 
first reaches zero, which corresponds to the plane where the surface charge is first neutralized by the ions, is denoted by $z_{\mathrm{NP}}$, the charge neutrality plane.

To quantify the distribution of the ions across the three layers as a function of the surface charge density, we calculate the cumulative charge density in the layers from an integral
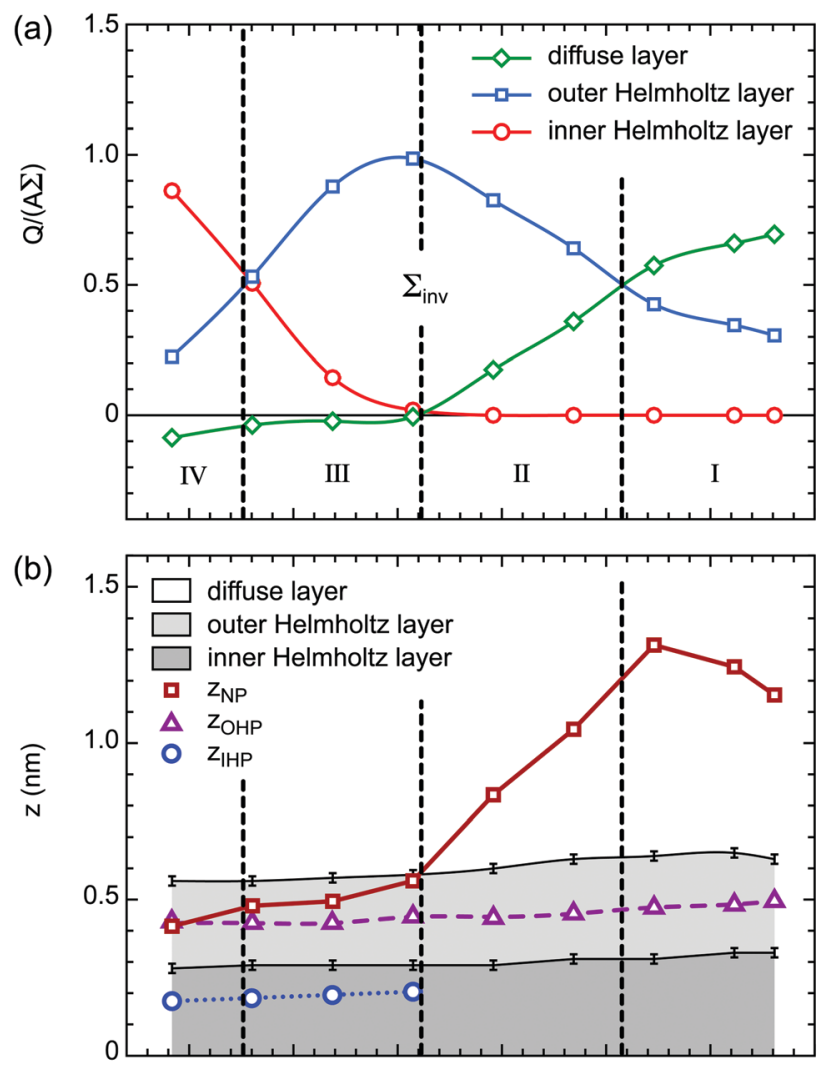

(c)

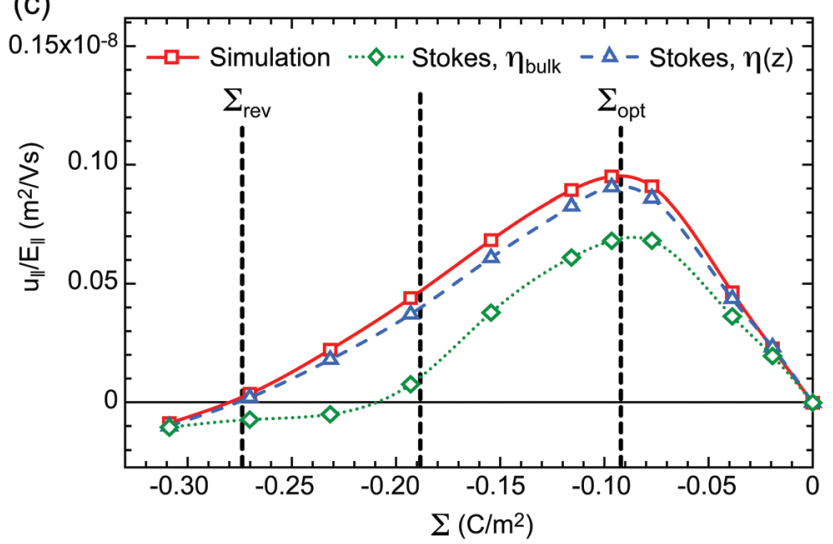

Fig. 3 (a) Cumulative charge in the electrolyte as a fraction of the surface charge, $Q /(A \Sigma)=\left(D_{\perp}\left(z_{i}\right)-D_{\perp}\left(z_{j}\right)\right) / \Sigma$, where $z_{i}$ and $z_{j}$ correspond to $z_{i}=z_{\mathrm{IHP}}$ and $z_{j}=0$ (red), $z_{i}=z_{\mathrm{OHP}}$ and $z_{j}=z_{\mathrm{IHP}}$ (blue) and $z_{i}$ equal to the channel center and $z_{j}=z_{\mathrm{OHP}}$ (green). (b) Positions of the neutral plane $z_{\mathrm{NP}}$, the outer and the inner Helmholtz planes (colored lines and symbols). Also shown are the boundaries of the outer Helmholtz layer $\left(\mathrm{z}_{\mathrm{OHL}}\right)$ and the inner Helmholtz layer $z_{\mathrm{IHL}}$ (black lines). (c) Electroosmotic velocity as a function of the surface charge density at a salt concentration of $1.7 \mathrm{M}$ and electric field of $0.55 \mathrm{~V} \mathrm{~nm}^{-1}$, using the parameters of Table 1 , with the solution to eqn (3) and (5) for $\eta(z)=\eta_{\text {bulk }}$ with $b=b_{\text {bulk }}$ (green diamonds) and for $\eta(z)$ shown in Fig. $4 \mathrm{~d}$ with $b=b_{\text {local }}$ (blue triangles). over the charge density (Fig. 3a). The integrated charge density gives rise to the definition of four different regimes. In regime I, more than half of the counter charge is located in the diffuse layer. In regime II, more charge accumulates in the outer Helmholtz layer, but the inner Helmholtz layer remains unpopulated. The surface charge where the inner Helmholtz layer starts acquiring a nonzero ion density coincides with the points at which the inner and outer Helmholtz layers together screen the entire surface charge and the diffuse layer is charge neutral. In regime III, the inner Helmholtz layer acquires an increasing number of counterions and the diffuse layer becomes negatively charged. Finally, in regime IV, the inner Helmholtz layer contains more than half of the counter charge. Importantly, even at the highest surface charge density investigated, the total charge in the inner Helmholtz layer alone never exceeds the surface charge.

The positions of the outer and inner Helmholtz planes are shown in Fig. 3b, together with the positions of the inner and outer Helmholtz layers (shaded regions) and the diffuse layer. The charge-neutral plane $z_{\mathrm{NP}}$ (shown by red squares) is located in the diffuse layer in regimes I and II and in the outer Helmholtz layer in regimes III and IV, showing that the Helmholtz layers carry more charge per unit area than the surface in these regimes. Note that this charge inversion occurs in the absence of non-electrostatic attraction, as can be seen from the purely repulsive potential of mean force at $\Sigma=0$ (see Section S5, ESI $\dagger$ ). Nevertheless, the details of the non-electrostatic repulsion will likely affect the point of charge inversion. Moreover, non-electrostatic interactions are highly ion and surface specific and could in general induce charge inversion in different systems.

\section{Electroosmotic mobility}

The effect of the charge inversion on the electroosmotic flow velocity is shown in Fig. 3c for the standard system (system 1, see Table S1, ESI $\dagger$ ). The electroosmotic velocity exhibits a nonmonotonous profile as a function of the surface charge density $\Sigma$, as reported previously. ${ }^{31-33}$ In particular, the electroosmotic flow exhibits a maximum when half of the charge is located in the Helmholtz layers, an inflection point appears when the surface charge is screened entirely in the Helmholtz layers, and mobility reversal is observed when the charge is primarily located in the inner Helmholtz layer. Based on these observations, we mark three special values of $\Sigma$ in Fig. 3: an optimum surface charge density at which the electroosmotic flow velocity reaches a maximum (denoted $\Sigma_{\text {opt }}$ ), the surface charge density at which charge inversion is observed $\left(\Sigma_{\text {inv }}\right)$, and the surface charge density at which the electroosmotic flow changes direction $\left(\Sigma_{\text {rev }}\right)$.

Table 1 Lennard-Jones (LJ) interaction parameters of the standard system (system 1, see Table S1, ESI). Geometric means, $\varepsilon_{\mathrm{ij}}=\sqrt{\varepsilon_{\mathrm{i}} \varepsilon_{\mathrm{j}}}$ and $\sigma_{\mathrm{ij}}=\sqrt{\sigma_{\mathrm{i}} \sigma_{\mathrm{j}}}$, are used to derive the parameters for dissimilar particles. The LJ cutoff radius is set to $1.1 \mathrm{~nm}$

\begin{tabular}{|c|c|c|c|c|c|}
\hline $\mathrm{i}$ & $\mathrm{H}$ & $\mathrm{O}$ & $\mathrm{Na}^{+}$ & $\mathrm{Cl}^{-}$ & $\mathrm{Si}$ \\
\hline$\varepsilon_{\mathrm{i}}\left(\mathrm{kcal} \mathrm{mol}^{-1}\right)$ & 0.0 & 0.155 & 0.0148 & 0.106 & 0.584 \\
\hline$\sigma_{\mathrm{i}}(\mathrm{nm})$ & 0.0 & 0.317 & 0.258 & 0.445 & 0.339 \\
\hline
\end{tabular}




\section{Effective hydrodynamic boundary condition}

Clearly, the surface charge densities of charge inversion and mobility reversal do not coincide. To find the origin of this discrepancy, we calculate the viscosity of the interfacial layers using a shear flow between two solid walls in the absence of an electric field (system 8, see Table S1, ESI $\dagger$ ). The nonlinearity of the velocity profiles (Fig. 4a) show that the viscosity differs from the bulk value in the interfacial layers. Additionally, at low surface charge density the fluid velocity at the surface differs from the wall velocity. Effectively, these two effects can be taken into account simultaneously by a homogeneous bulk viscosity combined with the Navier boundary condition,

$$
\left.b \frac{\mathrm{d} u_{\|}}{\mathrm{d} z}\right|_{0}=u_{\|}(0)
$$

for positive slip length $b$. In the case of a stagnant layer, $b$ becomes negative, and the velocity is nonzero only for $z>-b$.

We calculate the homogeneous bulk value, $\eta_{\text {bulk }}$, in separate simulations in the absence of the solid walls using the GreenKubo formulation, ${ }^{34}$

$$
\eta_{\text {bulk }}=\frac{V}{10 k_{\mathrm{B}} T} \int_{0}^{\infty}\left\langle\sum_{\alpha \beta} P_{\alpha \beta}\left(\tau+\tau_{0}\right) P_{\alpha \beta}\left(\tau_{0}\right)\right\rangle_{\tau_{0}} \mathrm{~d} \tau,
$$

with $P_{\alpha \beta}$ being the components of the stress tensor. The bulk viscosity of the electrolyte depends on the salt concentration $c_{\text {bulk }}$ (inset of Fig. $4 \mathrm{a}$ ), which in our shear and electroosmosis simulations depends slightly on the surface charge density. The bulk viscosity provides a good fit to the shear velocity in the center of the channel (Fig. 4a). By extrapolating the bulk profile to the wall velocity (Fig. 4b), we extract the effective bulk slip

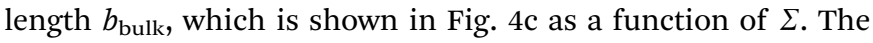
bulk slip length decreases sharply as a function of $\Sigma$, and turns into an effectively stagnant layer already at $\Sigma=-0.1 \mathrm{C} \mathrm{m}^{-2}$.

Next, we solve the Stokes equation using the simulated ionic charge density $e\left(c_{+}(z)-c_{-}(z)\right)$ as input. The Stokes equation for the velocity $u_{\|}(z)$ in the absence of a pressure gradient is given by

$$
0=\frac{\mathrm{d}}{\mathrm{d} z} \eta(z) \frac{\mathrm{d}}{\mathrm{d} z} u_{\|}(z)+E_{\|} e\left(c_{+}(z)-c_{-}(z)\right)
$$

with $\eta(z)$ being the viscosity of the fluid. The electroosmotic mobility calculated by eqn (5) using the effective hydrodynamic model with $\eta(z)=\eta_{\text {bulk }}$ and eqn (3) with $b=b_{\text {bulk }}$ (green diamond symbols in Fig. 3c) fails to capture the simulated electroosmotic mobility. In fact, mobility reversal is predicted at significantly lower $\Sigma$ than the one observed in the simulations. This shows that the effective description with a bulk viscosity and (a)

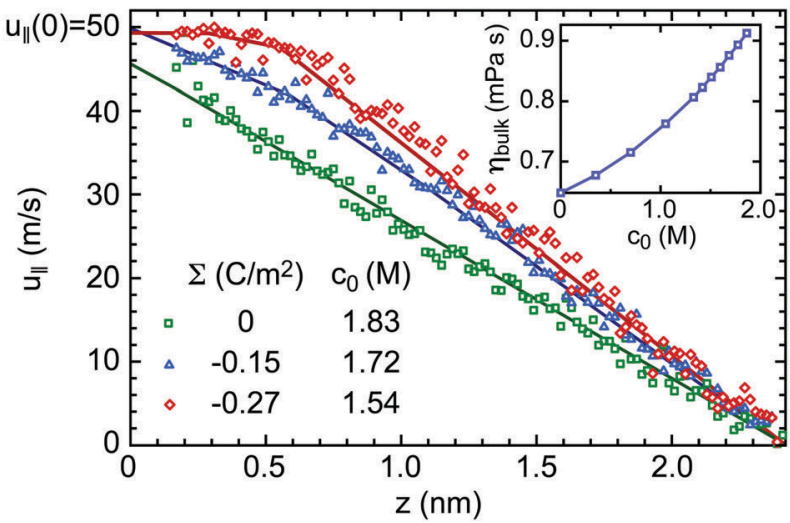

(b)

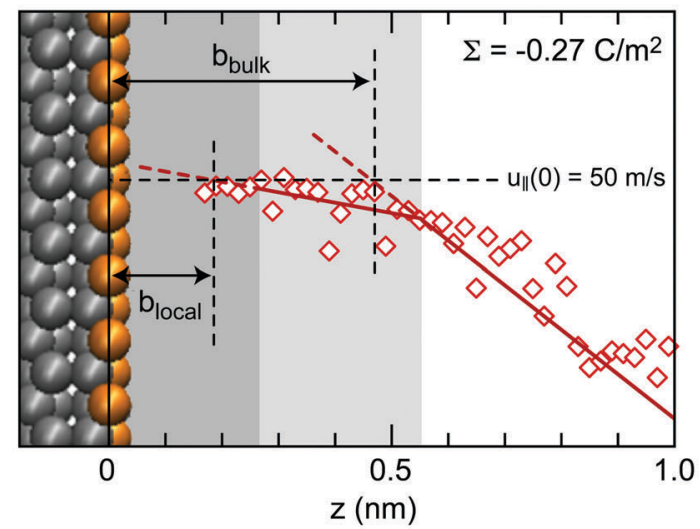

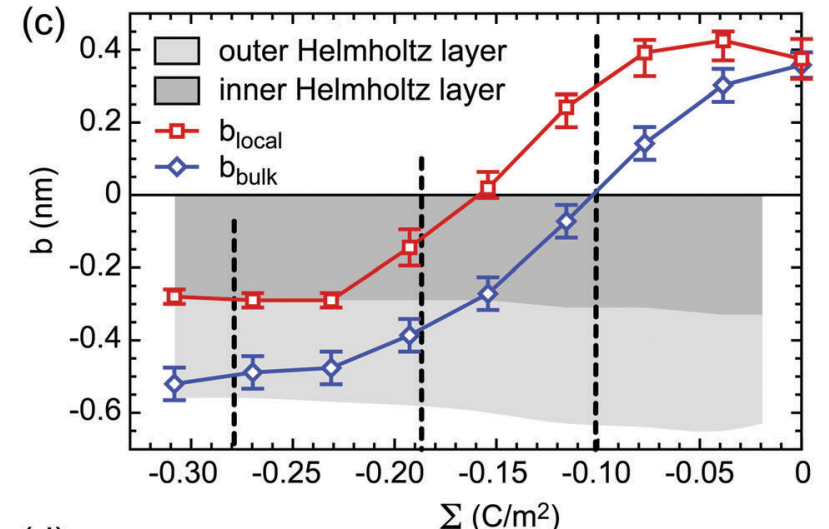

(d)

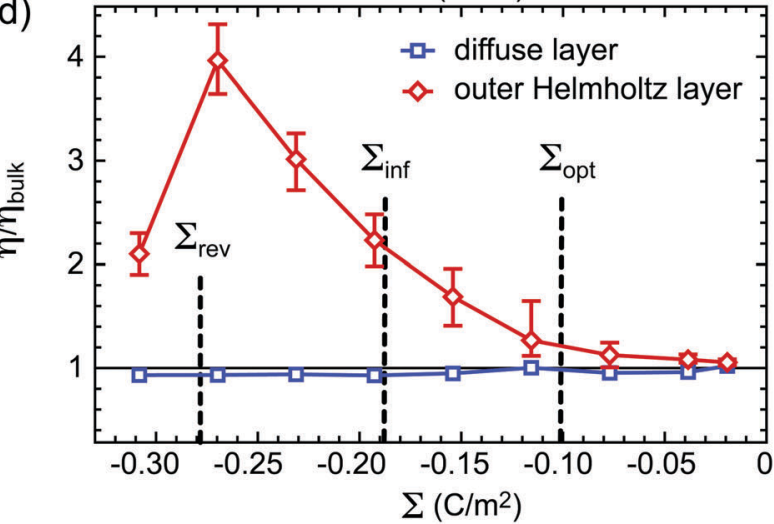

Fig. 4 (a) The shear velocity profile $u_{\|}(z)$ at a shearing velocity $u_{\|}(0)=50 \mathrm{~m} \mathrm{~s}^{-1}$ for different values of the surface charge density $\Sigma$, together with the piecewise linear fits. Curves are shown up to the center of the channel. The inset shows the bulk viscosity $\eta_{\text {bulk }}$ as a function of the salt concentration $c_{0}$ calculated in separate bulk simulations. (b) Magnification of (a) showing the piecewise linear fit and the definition of the slip lengths $b_{\text {local }}$ and $b_{\text {bulk. The }}$ shaded areas show the inner and outer Helmholtz layers. (c) The slip lengths defined in (b) as a function of $\Sigma$. (d) The resultant viscosities of the diffuse layer and the outer Helmholtz layer as a function of the surface charge density. The inner Helmholtz layer is stagnant within numerical error. 
the Navier boundary condition is not sufficient to reproduce the electroosmotic mobility as a function of the surface charge density.

\section{Interfacial layer viscosity}

To improve the description of the interfacial dynamics, we calculate the viscosity of the individual interfacial layers, instead of using the bulk viscosity with an effective Navier boundary condition. We use a piecewise linear fit to calculate the average viscosity of the different layers from $\eta(z)=-F /\left(A \mathrm{~d} u_{\|} / \mathrm{d} z\right)$, with $F$ being the friction force on the wall and $A$ being the surface area. $^{35}$ The viscosities of the outer Helmholtz layer and the diffuse layer are shown in Fig. 4d, showing a sharp increase of the outer Helmholtz layer viscosity with increasing $\Sigma$. The viscosity of the outer Helmholtz layer shows a sudden reduction at high surface charge density, which may be caused by the decreasing ion density in the outer Helmholtz layer. However, the decrease of the outer Helmholtz layer ion density starts already at $\Sigma=-0.2 \mathrm{C} \mathrm{m}^{-2}$ (Fig. 3a), showing that the ion density is not the only parameter affecting the local viscosity. In the cases where an inner Helmholtz layer is present, the velocity gradient in the inner Helmholtz layer is zero within numerical error, corresponding to infinite viscosity. In addition to $\eta(z)$, the system exhibits surface slip (Fig. 4a). This surface slip is taken into account using eqn (3), now using the first nonzero velocity gradient, given by $\mathrm{d} u_{\|} / \mathrm{d} z$ in the outer Helmholtz layer. This procedure gives rise to the definition of the local slip length $b_{\text {local }}$ (see Fig. $4 \mathrm{~b}$ for the graphical interpretation). Also $b_{\text {local }}$ decreases with $\Sigma$, but there is still a positive slip observed up to $\Sigma=-0.15 \mathrm{C} \mathrm{m}^{-2}$. Combining the viscosity profile $\eta(z)$ with $b_{\text {local }}$ reproduces the electroosmotic mobility profile as a function of $\Sigma$ very well (blue triangles in Fig. 3c), including the mobility reversal point $\Sigma_{\text {rev }}$. This shows that the formation of a viscous outer Helmholtz layer in combination with a local slip boundary condition is the key factor determining the surface charge density at which mobility reversal takes place, and charge inversion cannot be inferred from mobility reversal alone.

\section{Surface charge density regimes}

The four regimes of surface charge density defined in Fig. 3 can now be summarized as follows.

(I) $0<|\Sigma|<\left|\Sigma_{\text {opt }}\right|$ : in the first regime, the electrostatic surface forces are not strong enough to specifically adsorb the attracted ions; this means that the inner Helmholtz layer is electrically neutral (Fig. 3a). Moreover, the net charge of the ions accumulated in the outer Helmholtz layer is less than half the surface charge. Therefore, most of the counterions are located in the diffuse layer, where the viscosity is close to the bulk value (Fig. 4d), leading to the usual increase of the electroosmotic mobility with increasing surface charge density. The saturation of the mobility when $\Sigma$ approaches $\Sigma_{\text {opt }}$ is caused by the ions accumulating in the outer Helmholtz layer, which can largely be explained by dielectric effects. ${ }^{6,36}$ In the first regime, charge inversion does not occur, so the charge-neutral plane $z_{\mathrm{NP}}$ is located in the diffuse layer, at the point where the charge density vanishes permanently within numerical error. The slip length goes down steeply, as reported previously, ${ }^{25}$ but remains positive.
(II) $\left|\Sigma_{\text {opt }}\right|<|\Sigma|<\left|\Sigma_{\text {inv }}\right|$ : in the second regime, the surface electrostatic forces are strong enough to store more than half of the surface charge in the outer Helmholtz layer but they are too weak yet to supply the energy required for the partial dehydration and specific adsorption of the ions (Fig. 3a). The viscosity of the outer Helmholtz layer increases sharply (Fig. 4d), leading to a negative apparent slip length $b_{\text {bulk }}$ (Fig. 4c). The actual slip between the surface and the liquid, quantified by the local slip length $b_{\text {local }}$ remains positive. Thus, actual surface slip is combined with an increased interfacial viscosity, leading to a negative apparent

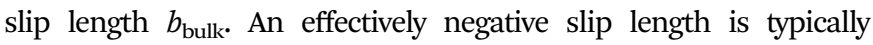
interpreted as indicative of a stagnant layer, but our results show that there is no stagnant layer in this regime.

(III) $\left|\Sigma_{\text {inv }}\right|<|\Sigma|<\left|\Sigma_{\text {rev }}\right|$ : When the surface charge density is high enough to give rise to an inner Helmholtz layer of adsorbed ions, the electroosmotic mobility exhibits an inflection point. The inner Helmholtz layer has a very high viscosity, making it effectively immobile with respect to the surface, which is reflected by the negative local slip length and therefore a truly stagnant layer. The viscosity of the outer Helmholtz layer continues to rise even though the total ion content of the outer Helmholtz layer starts decreasing (Fig. 3a and 4d). The neutral plane now lies inside the outer Helmholtz layer, and the total charge in the diffuse layer has changed sign. If both Helmholtz layers would be stagnant, $\Sigma_{\text {inv }}$ would be the point at which mobility reversal is expected, which is clearly not observed.

(IV) $\left|\Sigma_{\text {rev }}\right|<|\Sigma|$ : finally, the reversed electroosmotic flow is observed in cases where most of the surface charge is neutralized by the specifically adsorbed ions (Fig. 3a). This reversed electroosmotic mobility is due to a combination of charge inversion between the Helmholtz layers and the diffuse layer, a stagnant inner Helmholtz layer and increased viscosity in the outer Helmholtz layer.

\section{Dependence on the interfacial parameters}

The charge density at which the charge in the Helmholtz layers completely screens the surface charge density can be derived by equating the chemical potential of ions in the bulk and in the Helmholtz layers. Following that procedure, the surface charge density at which charge inversion takes place is expected to scale as $\left|\Sigma_{\text {inv }}\right|=2 r q c_{\text {bulk }} \exp \left[-\Delta \mu\left(r, q, c_{\text {bulk }}\right)\right]$, with $r$ being a length scale of the order of the counterion radius, $q$ being the counterion charge and $\Delta \mu\left(r, q, c_{\text {bulk }}\right)$ being an ionic free energy difference caused by correlation effects. ${ }^{18,37}$ In Fig. 5a, we show the dependence of the surface charge densities of charge inversion and mobility reversal as a function of the salt concentration $c_{\text {bulk }}$. The charge inversion charge density $\left|\Sigma_{\text {inv }}\right|$ decreases as a function of $N_{\mathrm{Cl}^{-}}$(which is proportional to $c_{\text {bulk }}$ ), showing that the dependence of the correlation free energy on the salt concentration is more important than the dependence of the prefactor on the salt concentration. Interestingly, for low concentration, charge inversion and mobility reversal coincide. At very low concentration $\left(N_{\mathrm{Cl}^{-}}=0\right.$, counterions only), charge inversion is not observed, as expected.

The dependence on the counterion size $\sigma_{\mathrm{Na}}$ is opposite for charge inversion and mobility reversal (Fig. 5b). This reflects the fact that counterion size has a strong effect on the free 

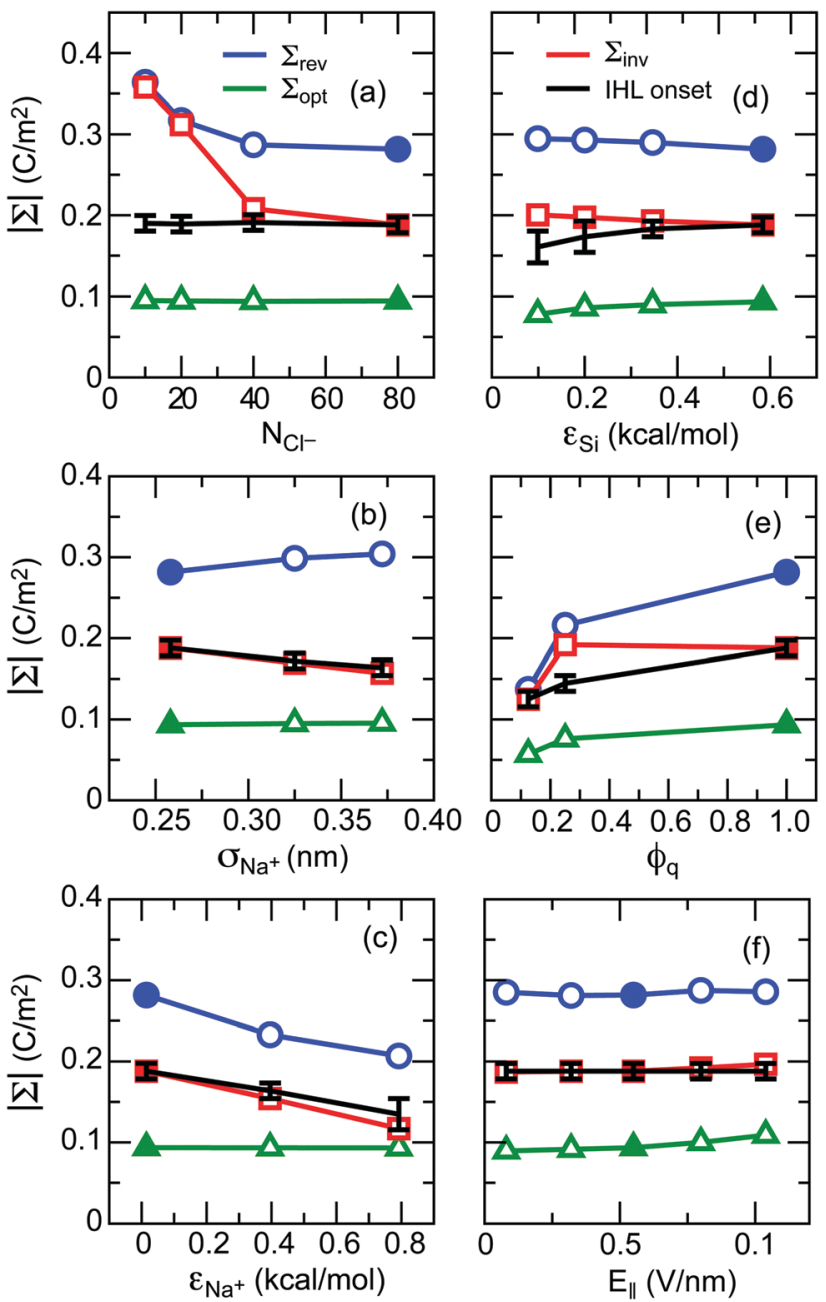

Fig. 5 The values of the absolute surface charge density $|\Sigma|$ at which charge inversion $\left(\Sigma_{\text {inv }}\right)$, mobility reversal $\left(\Sigma_{\text {rev }}\right)$ and the optimum in the electroosmotic mobility $\left(\Sigma_{\text {opt }}\right)$ take place as a function of the system parameters: (a) bulk salt concentration, parameterized by the number of coions $\mathrm{N}_{\mathrm{Cl}^{-}}$; (b) counterion van der Waals interaction size $\sigma_{\mathrm{Na}^{+}}$; (c) counterion van der Waals interaction strength $\varepsilon_{\mathrm{Na}^{+}}$; (d) surface van der Waals interaction strength $\varepsilon_{\mathrm{Si}}$; (e) the fraction of the surface atoms which are negatively charged $\phi_{q}$; and (f) the applied electric field strength $E_{\|}$. Results for the standard system (system 1, see Table S1, ESI $\dagger$ ) are indicated by the solid symbols. Also shown are the values of $|\Sigma|$ between which the inner Helmholtz layer first appears

energy of adsorption, which goes down with ion size, but bigger ions do not affect the viscosity as much as smaller ions. ${ }^{38}$ Apart from the reversal surface charge densities, increasing the counterion radius $\sigma_{\mathrm{Na}^{+}}$increases the magnitude of the mobility (see Fig. S2c, ESI $\dagger$ ).

Increasing the counterion interaction strength $\varepsilon_{\mathrm{Na}^{+}}$leads to a decrease of the inversion values of $\Sigma$ (Fig. 5c). By increasing $\varepsilon_{\mathrm{Na}^{+}}$, we increase the number of water molecules participating in the hydration shell of the cation. Evidence from quantum chemistry calculations suggests that a larger number of water molecules in the hydration shell decreases the dehydration energy, ${ }^{39,40}$ which could explain the reduced values of the charge densities of charge inversion and mobility reversal.
The interaction strength of the surface, $\varepsilon_{\mathrm{Si}}$, on the other hand, has only a minor effect on charge inversion (Fig. 5d). We attribute this to the fact that $\varepsilon_{\mathrm{Si}}$ does not affect the interfacial viscosity, nor the hydration properties, because it modifies the water-surface and the ion-surface interactions equally. However, increasing the wall interaction strength, which is known to reduce the surface slip, reduces the amplitude of the electroosmotic mobility. Note that in order to study the effect of the non-electrostatic interaction potential in more detail, the ionsurface and water-surface interactions will have to be varied independently.

We also verify the effect of the lateral distribution of the surface charges, quantified by the fraction $\phi_{q}$ of surface atoms which are charged, and find that a sparse distribution more readily induces charge inversion (Fig. 5e), in agreement with literature results. ${ }^{37}$ Decreasing the surface coverage to 1 in 4 atoms decreases the reversal charge density by a factor 2 . Moreover, reducing $\phi_{q}$ dramatically reduces the electroosmotic mobility. This shows that the surface roughness and inhomogeneity is a very important factor determining the charge reversal properties of charged solutes in water.

Finally, we check the linearity of the electrokinetic response as a function of the strength of the applied electric field. The electric field strength has no effect on the calculated $\Sigma$ values (Fig. 5f). Note, however, that as a function of the electric field strength, the electroosmotic mobility increases, which is caused by extension of the double layer width under influence of the hydrodynamic lift force. ${ }^{32}$ This nonlinear effect is not expected to be observed in experiments, because the electric field strength used is typically significantly lower. Note that the electric field strength used in this study is just outside the linear electroosmosis regime (see Fig. S2a, ESI $\dagger$ ). Nevertheless, the points of charge inversion, mobility reversal, electroosmotic optimum and onset of the inner Helmholtz layer are independent of the electric field, and the linear equations used in this work still capture the dynamics accurately.

At high salt concentration, charge inversion $\left(\Sigma_{\text {inv }}\right)$ coincides with the onset of the inner Helmholtz layer (black lines in Fig. 5). This can be rationalized by the decrease of the dielectric constant caused by the ions' loss of hydration shell when they form the inner Helmholtz layer, further enhancing the effect of the ion-ion correlations. The optimum surface charge for electroosmotic flow, $\Sigma_{\text {opt }}$, depends mildly on the surface properties $\varepsilon_{\mathrm{Si}}$ and $\phi_{q}$, but not on any other system parameters.

\section{Conclusions}

Our molecular dynamics simulations confirm the three-layer structure of the electric double layer proposed originally by Grahame, and show that with increasing surface charge density, ions first accumulate in the outer Helmholtz layer, before losing their hydration shell and forming an inner Helmholtz layer. For monovalent ions at a salt concentration of $1.7 \mathrm{M}$, charge inversion of the combined inner and outer Helmholtz layers occurs at $\Sigma=-0.19 \mathrm{C} \mathrm{m}^{-2}$, but the electroosmotic mobility does 
not show inversion up to $\Sigma=-0.27 \mathrm{C} \mathrm{m}^{-2}$. Using shear simulations, we show that the reason for this discrepancy is that whereas the inner Helmholtz layer is truly stagnant, the outer Helmholtz layer has a finite viscosity, which increases sharply with surface charge density. For an accurate description of the electroosmotic flow, and thus for a correct estimate of the charge inversion point from electrokinetic measurements, the description of the system in terms of a bulk viscosity and an effective Navier boundary condition is not enough. Instead, the combination of a local slip boundary condition with a space dependent viscosity profile $\eta(z)$ accurately reproduces the simulated electroosmotic velocity. We show that the system parameters such as the ion size and concentration, ion, surface and water interaction parameters and surface charge coverage affect the charge inversion process, as well as the electroosmotic mobility amplitude in nontrivial ways. Apart from providing an improved understanding of the dynamics of highly charged solutes, the dependence of the electrophoretic mobility and interfacial viscosity on the surface charge and other system parameters can help the design of technological applications such as foams, suspensions and coating flows.

\section{Conflicts of interest}

There are no conflicts to declare.

\section{Acknowledgements}

The Sheikh-Bahaei National High-performance Computing Center of Isfahan University of Technology, Iran, is acknowledged for their computational support.

\section{References}

1 H. Helmholtz, Ann. Phys., 1879, 243, 337.

2 M. Gouy, J. Phys. Theor. Appl., 1910, 9, 457.

3 D. L. Chapman, Philos. Mag., 1913, 25, 475.

4 O. Stern, Z. Elektrochem. Angew. Phys. Chem., 1924, 30, 508.

5 D. C. Grahame, Chem. Rev., 1947, 41, 441.

6 D. J. Bonthuis and R. R. Netz, J. Phys. Chem. B, 2013, 117, 11397.

7 N. C. Stellwagen, Electrophoresis, 2009, 30, S188.

8 A. V. Delgado, F. González-Caballero, R. J. Hunter, L. K. Koopal and J. Lyklema, J. Colloid Interface Sci., 2007, 309, 194.

9 I. Llorente, S. Fajardo and J. M. Bastidas, J. Solid State Electrochem., 2014, 18, 293.

10 D. J. Bonthuis and R. Golestanian, Phys. Rev. Lett., 2014, 113, 148101.

11 M. J. Pikal, Adv. Drug Delivery Rev., 2001, 46, 281.

12 Y. Uematsu, R. R. Netz and D. J. Bonthuis, Chem. Phys. Lett., 2017, 670, 11.
13 Y. Uematsu, R. R. Netz and D. J. Bonthuis, Langmuir, 2018, 34, 9097.

14 D. J. Barlow and J. M. Thornton, Biopolymers, 1986, 25, 1717.

15 B. Halle and M. Davidovic, Proc. Natl. Acad. Sci. U. S. A., 2003, 100, 12135.

16 A. Y. Grosberg, T. T. Nguyen and B. I. Shklovskii, Rev. Mod. Phys., 2002, 74, 329.

17 B. D. Storey and M. Z. Bazant, Phys. Rev. E: Stat., Nonlinear, Soft Matter Phys., 2012, 86, 056303.

18 K. Besteman, M. A. G. Zevenbergen and S. G. Lemay, Phys. Rev. E: Stat., Nonlinear, Soft Matter Phys., 2005, 72, 061501.

19 C. Calero, J. Faraudo and D. Bastos-González, J. Am. Chem. Soc., 2011, 133, 15025.

20 F. Vereda, A. Martín-Molina, R. Hidalgo-Alvarez and M. QuesadaPérez, Phys. Chem. Chem. Phys., 2015, 17, 17069.

21 M. Lidón López, M. Queralt-Martín and A. Alcaraz, Electrochem. Commun., 2014, 48, 32.

22 C. Schneider, M. Hanisch, B. Wedel, A. Jusufi and M. Ballauff, J. Colloid Interface Sci., 2011, 358, 62.

23 I. Semenov, S. Raafatnia, M. Sega, V. Lobaskin, C. Holm and F. Kremer, Phys. Rev. E: Stat., Nonlinear, Soft Matter Phys., 2013, 87, 022302.

24 J.-L. Barrat and L. Bocquet, Phys. Rev. Lett., 1999, 82, 4671. 25 L. Joly, C. Ybert, E. Trizac and L. Bocquet, J. Chem. Phys., 2006, 125, 204716.

26 H. J. C. Berendsen, J. R. Grigera and T. P. Straatsma, J. Phys. Chem., 1987, 91, 6269.

27 J. Eastwood, R. Hockney and D. Lawrence, Comput. Phys. Commun., 1980, 19, 215.

28 S. Plimpton, J. Comput. Phys., 1995, 117, 1.

29 N. P. Brandon and G. H. Kelsall, J. Appl. Electrochem., 1985, 15, 485.

30 Y. Uematsu, D. J. Bonthuis and R. R. Netz, J. Phys. Chem. Lett., 2018, 9, 189.

31 L. Joly, F. Detcheverry and A.-L. Biance, Phys. Rev. Lett., 2014, 113, 088301.

32 M. Rezaei, A. Azimian and D. Toghraie, Phys. A, 2015, 426, 25.

33 M. Rezaei, A. Azimian and D. Toghraei, Heat Mass Transfer, 2015, 51, 661.

34 P. J. Daivis and D. J. Evans, J. Chem. Phys., 1994, 100, 541.

35 A. Schlaich, J. Kappler and R. R. Netz, Nano Lett., 2017, 17, 5969.

36 D. J. Bonthuis and R. R. Netz, Langmuir, 2012, 28, 16049.

37 J. Faraudo and A. Travesset, J. Phys. Chem. C, 2007, 111, 987.

38 D. E. Goldsack and R. Franchetto, Can. J. Chem., 1977, 55, 1062.

39 J. A. Lopez-Berganza, Y. Diao, S. Pamidighantam and R. M. Espinosa-Marzal, J. Phys. Chem. A, 2015, 119, 11591.

40 S. Inaba and W. M. C. Sameera, J. Phys. Chem. A, 2016, 120, 6670. 